\title{
Communication
}

\section{Evidence for the presence of the Kennedy and Bremer- Greenberg pathways in Caenorhabditis elegans}

\author{
Günter Lochnit and Rudolf Geyer ${ }^{\varpi}$ \\ Institute of Biochemistry, Faculty of Medicine, University of Giessen, \\ D-35392 Giessen, Germany
}

Received: 22 July 2003; accepted 20 August 2003

Key words: nematodes; choline metabolism; phosphatidylcholine biosynthesis

\begin{abstract}
Nematodes were found to synthesize phosphorylcholine-containing molecules not present in higher organisms, i.e. phosphorylcholine-substituted glycosphingolipids and (glyco)proteins. Investigations on the biosynthesis of these structures provided first biochemical evidence for the presence of the Kennedy and Bremer-Greenberg pathways in the model organism Caenorhabditis elegans.
\end{abstract}

Nematodes can be divided into plant- and animal-parasitic as well as free living species. Due to their life cycles, they have to adopt to different environmental conditions. Since the cuticle of parasitic nematodes is the phospholipid-rich interface of these organisms with their environment, knowledge on the composition and biosynthesis of this structure might help to understand their resistance towards environmental changes
(Smith et al., 1996). In addition to the cholinebearing molecules found in vertebrates, e.g. phosphatidylcholine (Ptd-Cho), sphingomyelin (SM), acetylcholine and platelet-activating factor (PAF) as well as their metabolic intermediates, nematodes contain phosphorylcholine ( $P$ Cho) and, in part, phosphorylethanolamine ( $P$ Etn)-substituted glycosphingolipids as well as PCho-conjugated (glyco)proteins (Lochnit et al., 2000). The glyco-

\footnotetext{
${ }^{-}$This study was supported by the German Research Council (SFB 535)

${ }^{\otimes}$ Corresponding author: Rudolf Geyer, Institute of Biochemistry, Faculty of Medicine, University of Giessen, Friedrichstrasse 24, D-35392 Giessen, Germany; tel: (49 641) 994 7400; fax: (49 641) 994 7409; E-mail: Rudolf.Geyer@biochemie.med.uni-giessen.de

Abbreviations: AHC, adenosylhomocysteine; DAG, diacylglyceol; TLC, thin-layer chromatography; PAF, platelet-activating factor; PCho, phosphorylcholine; PEtn, phosphorylethanolamine; Ptd-Cho, phosphatidylcholine; Ptd-Etn, phosphatidylethanolamine; Ptd-Serine, phosphatidylserine; SAM, $S$-adenosylmethionine; SM, sphingomyelin.
} 
sphingolipid bound PCho-epitopes can be regarded as phylogenetic markers of nematodes (Wuhrer et al., 2000). Furthermore, $P C h o-s u b s t i t u t e d$ antigens were found to be potent immunomodulatory molecules (Harnett \& Harnett, 1999; 2001). Whereas the biosynthesis of Ptd-Cho in mammals is quite well understood (Vance, 1989), that of the glycosphingolipid- and (glyco)protein-based $P C h o-e p i t o p e s$ in nematodes remains unclear. Neither the donor for the PCho-substituents nor the involved transferases have been identified so far, although the transfer of PCho to enzymatic methylation using $S$-adenosylmethionine (SAM) as methyl group donor (Bremer-Greenberg pathway). Ptd-Etn can be formed either via ethanolamine, PEtn and CDP-Etn or by decarboxylation of phosphatidylserine (Ptd-Serine; see Fig. 1).

Amongst the helminths (nematodes, cestodes and trematodes), the presence of both pathways has been demonstrated for the filariid Dirofilaria immitis (Srivastava \& Jaffe, 1985), whereas only the Kennedy pathway was found in the parasitic trematodes Fasciola hepatica and Schistosoma mansoni

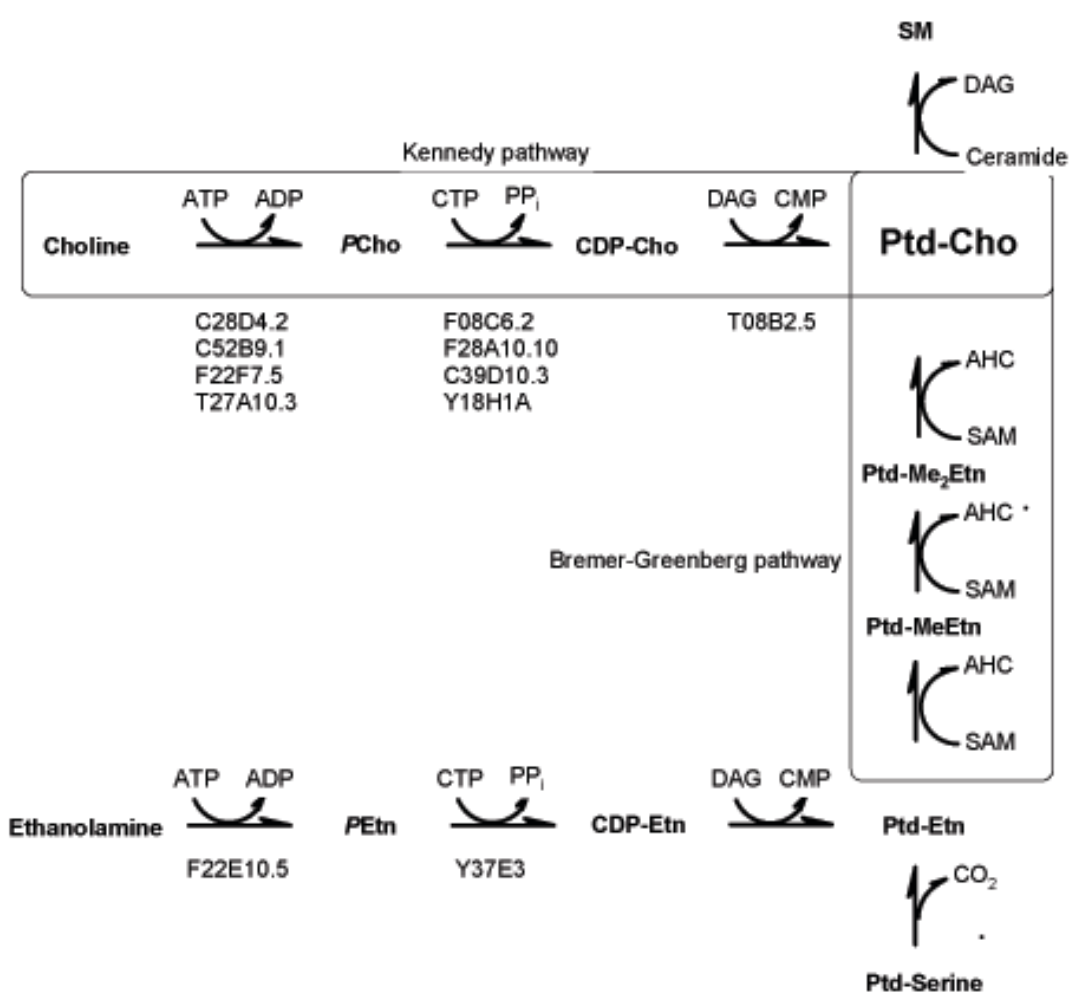

Figure 1. Kennedy and Bremer-Greenberg pathways.

Open reading frames of putative enzymes from C. elegans are indicated. AHC, adenosylhomocysteine; DAG, diacylglycerol.

glycosphingolipids and (glyco)proteins might represent a novel target for chemotherapy against these parasites.

For the formation of Ptd-Cho, two biosynthetic routes have been reported to exist: (a) the incorporation of choline via PCho and CDP-choline (Kennedy pathway) and (b) the conversion of Ptd-Etn to Ptd-Cho by stepwise
(Oldenberg et al., 1975; Young \& Podesta, 1982). The genome of the model nematode $C$. elegans contains, based on homology search, several putative genes for the enzymes of the Kennedy and Bremer-Greenberg pathway (see Fig. 1; http://www.wormbase.org). Investigations on the phospholipids of C. elegans, however, have been focused in the 
past mainly on their fatty acid compositions (Satouchi et al., 1993; Tanaka et al., 1999), whereas studies on the biosynthesis of these compounds have been restricted, so far, to cloning and characterization of a CTP:phosphorylcholine cytidylyltransferase from this worm (Friesen et al., 2001).

\section{MATERIALS AND METHODS}

Axenic cultivation of C. elegans. For biosynthetic studies we established an axenic culture of C. elegans (Vanfleteren, 1978). Cultivated in a completely chemically defined medium, the worms need approx. 6-7 days to complete their life cycle.

Metabolic labelling of $\mathrm{C}$. elegans. Worms were cultivated for 7 days in the presence of [methyl $\left.-{ }^{14} \mathrm{C}\right]$ choline, $\quad\left[\right.$ methyl $\left.-{ }^{14} \mathrm{C}\right] P C h o$, [methyl- $\left.{ }^{14} \mathrm{C}\right] \mathrm{CDP}-\mathrm{choline}$ or $\left[\right.$ methyl $\left.-{ }^{14} \mathrm{C}\right] \mathrm{SAM}$ ( $10^{6}$ c.p.m. $/ \mathrm{ml}$ each), harvested and homogenized. A two-step, phase-separation protocol was used for isolation and analysis of metabolic intermediates (Houston et al., 2002) which included a Bligh and Dyer partition (Bligh \& Dyer, 1959) followed by a butanol extraction of the dried methanol/water phase. Radiolabelled phospholipids were recovered in the chloroform phases.

Isolation of microsomal fractions. To further characterize enzymes involved in the Kennedy pathway, we prepared microsomal fractions from $C$. elegans. Worms were homogenized in GTE-buffer (20\% glycerol, 50 $\mathrm{mM}$ Tris/HCl, $\mathrm{pH}$ 7.4, $1 \mathrm{mM}$ EDTA) and the extract was centrifugated at $16000 \times \boldsymbol{g}$ for 10 min. The supernatant was further centrifugated at $130000 \times \mathbf{g}$ for $45 \mathrm{~min}$ and the microsomal pellet was resuspended in GTE buffer.

Cholinephosphotransferase assay. For the cholinephosphotransferase assay, $20 \mu \mathrm{l}$ of microsomal suspension (containing $100 \mu \mathrm{g}$ protein) was added to $20 \mu \mathrm{l}$ of $50 \mathrm{mM}$ Tris/ $\mathrm{HCl}, \mathrm{pH} 8.5$, containing $10 \mathrm{mM} \mathrm{MgCl}_{2}$, $0.5 \mathrm{mM}$ EGTA, $2.4 \mathrm{mM}$ sn-1,2-diolein and
22000 c.p.m. $\left[{ }^{14} \mathrm{C}\right] \mathrm{CDP}$-choline (Cornell, 1992). After $15 \mathrm{~min}$ at $37^{\circ} \mathrm{C}$ the reaction was stopped by adding $1.5 \mathrm{ml}$ methanol/chloroform $(2: 1, \mathrm{v} / \mathrm{v})$ and the reaction mixture was subjected to a Bligh and Dyer phase separation (Bligh \& Dyer, 1959).

\section{RESULTS AND DISCUSSION}

C. elegans was cultivated in axenic medium. In contrast to monoxenic cultures with Escherichia coli as food source, this cultivation avoids the presence of a metabolically interfering organism. Results from feeding and inhibition experiments can, therefore, be directly attributed to C. elegans.

Worms were cultivated for 7 days in the presence of the radiolabelled precursors $\left[{ }^{14} \mathrm{C}\right]$ choline, $\left[{ }^{14} \mathrm{C}\right]$ PCho, $\left[{ }^{14} \mathrm{C}\right] \mathrm{CDP}$-choline or $\left[{ }^{14} \mathrm{C}\right] \mathrm{SAM}$, harvested, homogenized and subjected to a two-step phase-separation protocol. TLC-separation of the metabolites and iodine staining revealed the presence of Ptd-Etn, Ptd-Cho and SM in the chloroform phase. Subsequent autoradiography revealed the presence of $\left[{ }^{14} \mathrm{C}\right]$ choline in Ptd-Cho and SM in all four labelling experiments (see Fig. 2) in comparable amounts, thus demonstrating the presence of both the Kennedy and the Bremer-Greenberg pathway in C. elegans.

To further characterize enzymes involved in the Kennedy pathway, we prepared microsomal fractions from $C$. elegans and performed a cholinephosphotransferase assay. Analysis of the products revealed the synthesis of radiolabelled phosphatidylcholine. The choline phosphotransferase activity could be inhibited by farnesol $(10-160 \mu \mathrm{M})$ in a dose-dependent manner to approx. $60 \%$ (see Fig. 3). This type of inhibition has been also reported for the mammalian cholinephosphotransferase (Miquel et al., 1998).

In conclusion, these data demonstrate that C. elegans can take up choline, PCho, CDP-Cho as well as SAM and can use these metabolites as substrates for Ptd-Cho bio- 


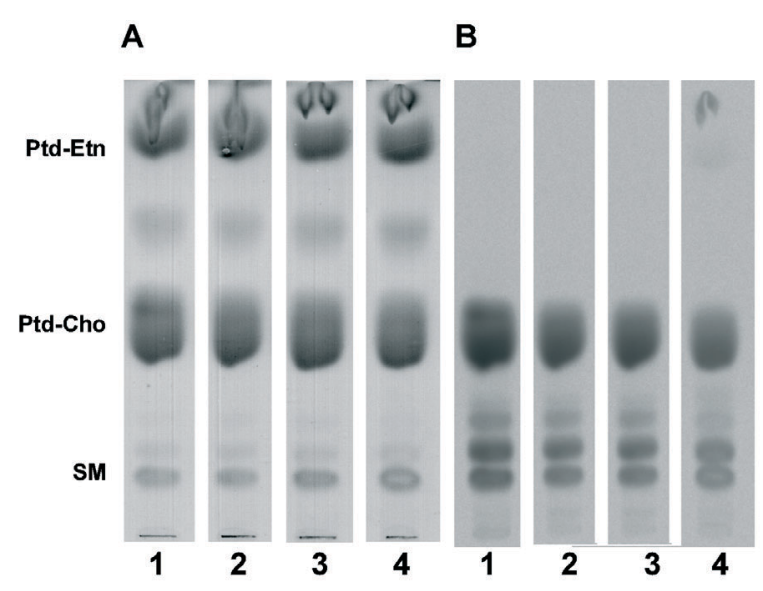

Figure 2. Analysis of radioactive labelled phospholipids from C. elegans.

Worms were cultivated in the presence of $\left[{ }^{14} \mathrm{C}\right]$ choline (lane 1), [ $\left.{ }^{14} \mathrm{C}\right] P C h o$ (lane 2), $\left[{ }^{14} \mathrm{C}\right] \mathrm{CDP}-\mathrm{Cho}$ (lane 3) or $\left[{ }^{14} \mathrm{C}\right] \mathrm{SAM}$ (lane 4). After phase separation, aliquots of the chloroform phase were separated on TLC-plates and phospholipids were visualized either by staining with iodine (A) or by autoradiography (B).

synthesis. The fact that SAM leads to the incorporation of radioactivity into Ptd-Cho in comparable amounts as the other labelled

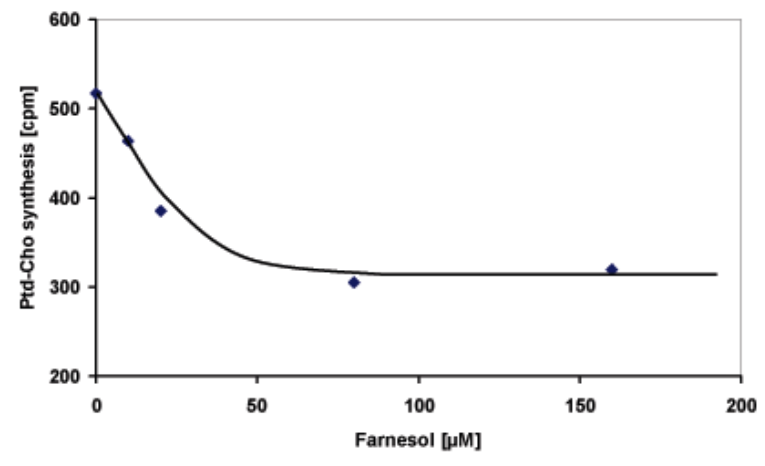

Figure 3. Inhibition of cholinephosphotransferase by farnesol in C. elegans microsomes.

compounds indicates that the BremerGreenberg pathway plays an important role in choline metabolism of this model organism. The presence of two biosynthetical pathways for the synthesis of Ptd-Cho might be advantageous for the nematodes by rendering the worms independent from the uptake of choline from the environment. Furthermore, this might explain, that RNA interference ex- periments targeting the cholinephosphotransferase (T08B2.5) did not show any phenotype (Fraser et al., 2000) in C. elegans. This model organism can, therefore, be also considered as an excellent system to study phospholipid biosynthesis and metabolism.

The authors thank F. Busch, M. Schwinn and D. Zimmer for excellent technical assistance.

\section{R E F E R E N C E S}

Bligh EG, Dyer WJ. (1959) Can J Biochem Physiol.; 37: 911-7.

Cornell RB. (1992) Methods Enzymol.; 209: 267-72.

Fraser AG, Kamath RS, Zipperlen P, Martinez-Campos M, Sohrmann M, Ahringer J. (2000) Nature.; 408: 325-30.

Friesen JA, Liu MF, Kent C. (2001) Biochim Biophys Acta.; 1533: 86-98.

Harnett W, Harnett MM. (1999) Immunol Today.; 20: 125-9.

Harnett W, Harnett MM. (2001) Biochim Biophys Acta.; 1539: 7-15.

Houston K, Lochnit G, Geyer R, Harnett W. (2002) Mol Biochem Parasitol.; 123: 55-66.

Lochnit G, Dennis RD, Geyer R. (2000) Biol Chem.; 381: 839-47.

Miquel K, Pradines A, Terce F, Selmi S, Favre G. (1998) J Biol Chem.; 273: 26179-86.

Oldenberg V, Vugt Fv, Golde LMGv. (1975) Biochim Biophys Acta.; 398: 101-10.

Satouchi K, Hirano K, Sakaguchi M, Takehara H, Matsuura F. (1993) Lipids.; 28: 837-40.

Smith VP, Selkirk ME, Gounaris K. (1996) Mol Biochem Parasitol.; 78: 105-16.

Srivastava AK, Jaffe JJ. (1985) Int J Parasitol.; 15: $27-31$. 
Tanaka T, Izuwa S, Tanaka K, Yamamoto D, Takimoto T, Matsuura F, Satouchi K. (1999) Eur J Biochem.; 263: 189-95.

Vance DE. (1989) Phosphatidylcholine metabolism. CRC Press, Boca Raton.
Wuhrer M, Rickhoff S, Dennis RD, Lochnit G, Soboslay PT, Baumeister S, Geyer R. (2000) Biochem J.; 348: 417-23.

Young BW, Podesta RB. (1982) Mol Biochem Parasitol.; 5: 165-72.

Vanfleteren JR. (1978) Ann Rev Phytopathol.; 16: $131-57$. 